\title{
Aspects physico-chimiques du nettoyage de surface par laser: critères de choix de la longueur d'onde
}

\author{
R. Oltra et J.P. Boquillon* \\ Laboratoire de Recherches sur la Réactivité des Solides, UMR 5613 du CNRS, \\ Université de Bourgogne, France \\ * Laboratoire de Physique, UPRES A 5027 du CNRS, Université de Bourgogne, France
}

\begin{abstract}
Résumé: Le procédé de décapage de surface par faisceau laser impulsionnel est actuellement envisagé comme une technique de substitution à des procédés de nettoyage chimique afin de réduire les risques sur l'environnement ou à des procédés mécaniques afin de réduire dans ce cas les risques d'endommagement du substrat à nettoyer.

Si les sources lasers choisies peuvent être regroupées dans le domaine des lasers impulsionnels (temps d'interaction de l'ordre de la nanoseconde), le choix de la longueur d'onde est rarement justifié à partir d'une étude physicochimique de l'interphase substrat-contaminant à expulser. Différents exemples sont présentés illustrant le besoin de développer des bases de données.
\end{abstract}

\section{INTRODUCTION}

Différentes applications des lasers impulsionnels ont été envisagées afin de remplacer des techniques chimiques ou bien mécaniques (abrasion par des particules submicroniques) de dépollution de surfaces contaminées par exemple par des films de graisse ou bien d'oxydes. L'objectif consiste à réduire les effets nocifs sur l'environnement dus en particulier à l'usage trop intensif de solvants comme le trichloroéthylène ou le CFC (CarbonFluoroChloride).

Différents domaines industriels ont été intéressés par ce type de technique:

- la micro-électronique, l'optique,

- les industries de transformation,

- le décapage de surface en milieu hostile.

Dans le domaine de la micro-électronique, il s'agit principalement d'utiliser les sources laser impulsionnelles pour expulser les particules submicroniques très adhérentes qui sont impossibles à éliminer par décapage ultra ou mégasoniques [1-4]

Les industries de transformation, comme les fabriquants de tubes métalliques ou de récipients en verre, s'intéressent à cette nouvelle technologie afin d'améliorer l'élimination de couches polluantes (huile de laminage) ou de contaminations résiduelles liées au procédé d'élaboration (film de silice lubrifiant les filières de verrerie). On peut également citer à titre d'exemple, le décapage de peintures dans l'industrie aéronautique qui permet de procéder régulièrement à des examens de contrôle des structures [5]. Tous ces traitements ont comme caractéristique commune, l'élimination de films minces en surface en limitant au mieux les risques d'endommagement du substrat.

\section{RESULTATS ET DISCUSSION}

\subsection{Principes physico-chimiques du nettoyage laser: les différents modes d'interaction}

Lorsqu'un faisceau laser impulsionnel (domaine ns voire ps) va interagir avec ce type d'interface (substratcontaminants) différents phénomènes physiques et chimiques peuvent se dérouler en fonction des caractéristiques du faisceau laser et de la nature même des produits susceptibles d'être éliminés. On peut regrouper ces différents types d'interaction selon trois types d'effets:

- effet thermique: il y a absorption directe de la lumière par le contaminant et celui-ci est éliminé par vaporisation et génération d'ondes de chocs, 
- effet photonique direct: il s'agit de processus photochimiques qui conduisent à la rupture des liaisons chimiques du contaminant qui sera ablaté et ionisé (principalement par interaction dans le domaine UV),

- effet photonique indirect: dans le cas de contaminants non absorbants, l'énergie laser est déposée à l'interface interne substrat-film contaminant. La relaxation des processus photoniques générés dans le substrat conduit, dans le cas de matériaux métalliques, à des déformations thermoélastiques qui provoquent la décohésion du film de contaminants (Figure1).

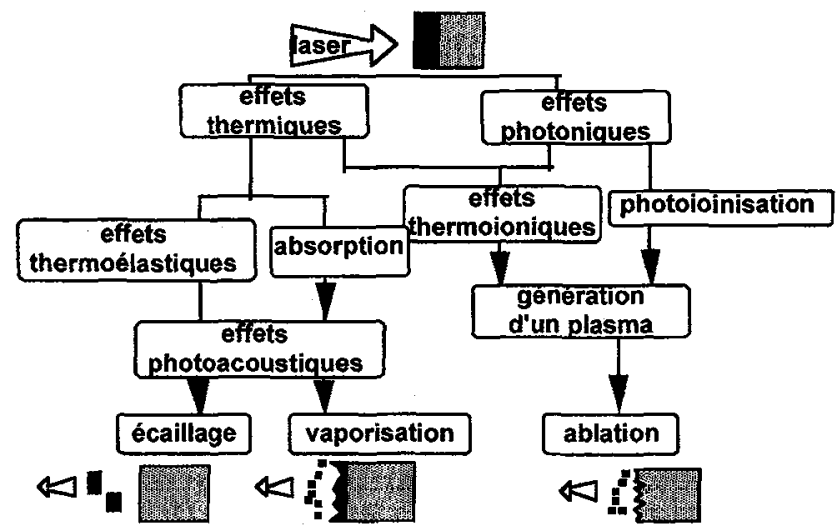

Figure 1- Représentation schématique des différents types d'interaction laser-film conduisant au nettoyage d'une surface recouverte d'un film mince de produits contaminants.

\subsection{Exemples de choix de longueur d'onde}

\subsubsection{Décontamination de surface en milieu liquide}

Le nettoyage de surfaces contaminées par des microparticules est un problème rencontré dans le domaine de la microélectronique. Des particules submicroniques venant du polissage, comme des particules d'alumine, peuvent par exemple endommager des têtes de lecture. Différents travaux ont été rapportés sur l'élimination par interaction laser de ces particules présentes à la surface de substrats comme le silicium [6]. Plus récemment un ensemble de travaux menés sur ce type de système (particules submicroniques) ont été réalisé en présence d'une phase liquide afin d'amplifier les effets mécaniques ou de mieux contrôler l'hydrodynamique [6-9].

Ces diverses investigations diffèrent principalement par le choix de la longueur d'onde qui va conditionner comme le montre la figure 2 le type d'interaction et donc le mode d'expulsion ainsi que les risques d'endommagement susceptibles d'affecter le substrat.

Selon la longueur d'onde on observe:

- pour la longueur d'onde $10.6 \mu \mathrm{m}$, l'absorption dans la phase liquide se produit sur des profondeurs de $10 \mu \mathrm{m}$ et on peut s'attendre à un échauffement homogène du film liquide suffisamment mince et donc à une vaporisation satisfaisante [6],

- pour une longueur d'onde correspondant à l'emploi d'un laser Er:YAG $(2.3 \mu \mathrm{m})$, une absorption sélective à la surface de la phase liquide (eau) qui conduit à une expulsion du film liquide entraînant partiellement les particules déposées à la surface du substrat [6],

- pour des longueurs d'ondes dans le visible (laser Nd:YAG doublé 532nm), il s'agit d'une absorption principalement de l'énergie laser à l'interface interne substrat - liquide et une absorption faible par les contaminants. Ceci conduit principalement à la vaporisation interne de la phase liquide à cause de l'échauffement transitoire qui crée des bulles entrainant les particules en surface. Dans ce cas il existe peu de risques de décomposition thermique des particules qui pourrait conduire à des modifications chimiques de la surface $[7,8]$. 
- pour des longueurs d'ondes dans l'UV (laser $\mathrm{KrF} 248 \mathrm{~nm}$ ou XeCl $308 \mathrm{~nm}$ ), le même type de phénomène que précédemment est observé avec cependant une contribution plus importante des particules elles-mêmes qui absorbent l'énergie laser et donc qui par leur propre déformation vont générer des forces qui vont s'opposer aux forces de Van der Walls qui créent leur adhésion [9].

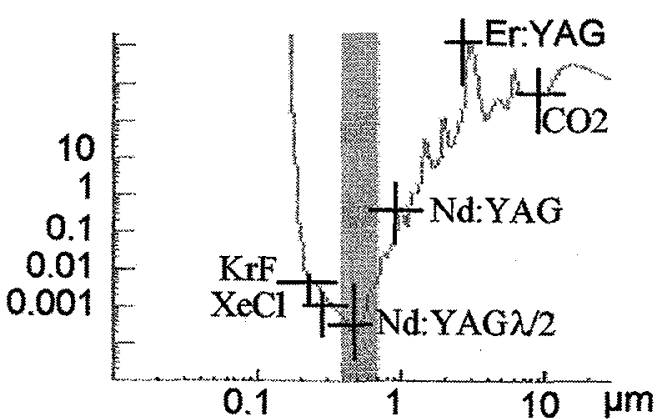

Figure 2- Spectre d'absorption de l'eau.

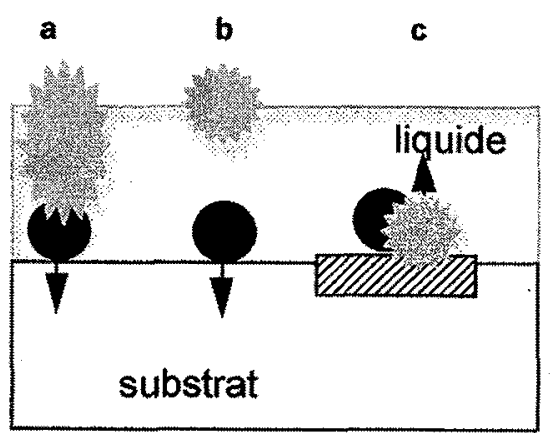

Figure 3- Schéma représentant le type d'interaction laser système substrat -particule - film de liquide

a) $\mathrm{CO} 2$, b) Er:YAG , c) Nd:YAG (532nm) et UV.

Les travaux les plus avancés dans ce domaine [8,9] tendent à privilégier les lasers dans le visible ou l'UV.

\subsubsection{Nettoyage de produits métalliques laminés ou étirés à froid}

Dans ce cas, l'objectif est d'éliminer une fine pellicule d'huile de la surface de tôles laminées ou de l'intérieur de tubes étirés. Cependant la nature même de l'huile «usagée» de laminage doit être prise en compte: il s'agit en fait d'une suspension de microparticules métalliques arrachées par micro-usinage lors du processus mécanique, en suspension dans le lubrifiant (Figure 4).

Dans l'étude sur les tubes, les auteurs ont utilisé un laser déclenché Nd:YAG $(1.06 \mu \mathrm{m})$ [10] alors que dans l'étude des tôles laminées les travaux ont été effectués dans le visible ou l'UV [11].

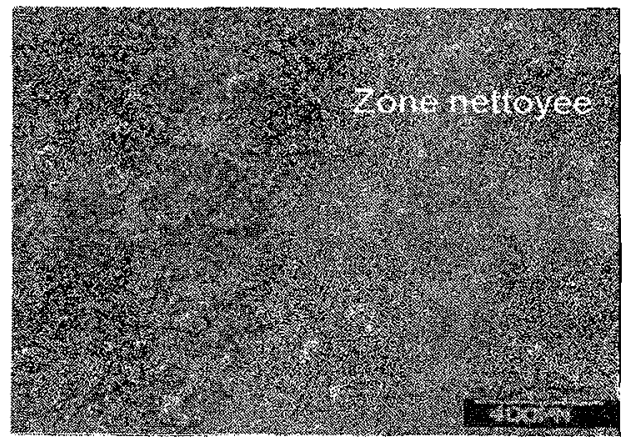

Figure 4 - Illustration du nettoyage laser : le film d'huile de laminage contenant de fines particules métalliques est visible sur la partie gauche, la zone décapée étant à droite [10].

Dans le cas des tubes [10], le spectre d'absorption de l'huile «vierge» montre que l'énergie laser est absorbée à $532 \mathrm{~nm}$ et pas à $1.06 \mu \mathrm{m}$ (Figure 5). En fonction de la longueur d'onde, on pourrait donc s'attendre à une différence bien claire des modes d'interactions. En fait les mesures de pertes de masse effectuées en temps réel sur des échantillons recouverts d'huile «usagée» montrent clairement que le rendement en termes d'efficacité ne dépend pas de la longueur d'onde choisie (Figure 6). 


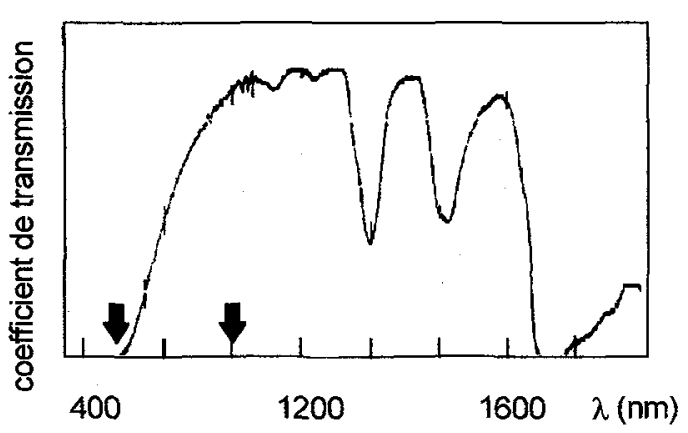

Figure 5 - Spectre d'absorption du lubrifiant «vierge» [10].

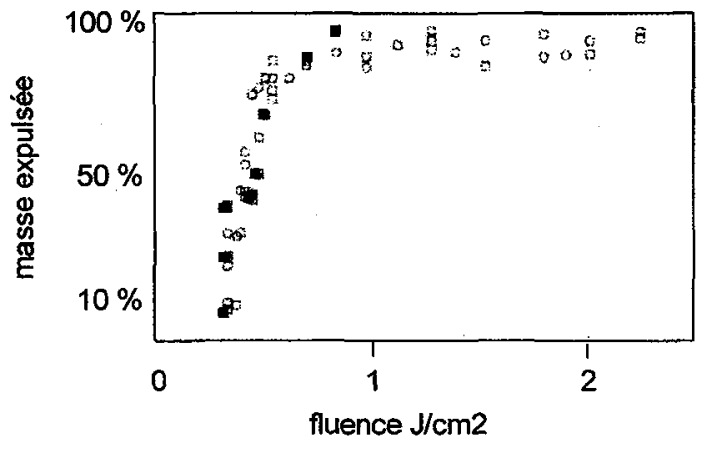

Figure 6 - Mesure de l'efficacité du décapage laser Nd:YAG $(1.06 \mu \mathrm{m})$ sur des tubes pollués nuance acier inoxydable $316 \mathrm{~L}$ (घ) $532 \mathrm{~nm}$ (口) $1064 \mathrm{~nm}$ [10].

Ceci peut s'expliquer par le fait que l'énergie laser est principalement absorbée par les particules en suspension plus que par le lubrifiant. La quantité d'huile expulsée a été déterminée par des mesures de masse en temps réel sur une microcrobalance (type SETARAM sensibilité $1 \mu \mathrm{g}$ ). On privilégie ainsi la contribution thermomécanique qui expulse les particules métalliques ainsi qu'une grande partie du volume d'huile sous forme de goutelettes. Par la suite, les traitements industriels ont donc été effectués à $1.06 \mu \mathrm{m}$ de façon à éviter toute forme d'interaction laser-lubrifiant afin de réduire les risques de décomposition chimique. Les analyses de surface (SIMS) confirment que les constituants de l'huile ne diffusent pas à l'intérieur du substrat métallique.

Dans le cas des tôles laminées, c'est au contraire le mode désorption qui a été privilégié en utilisant un laser $\mathrm{KrF}(193 \mathrm{~nm})$, ce traitement photochimique pouvant être complété par des tirs laser à $532 \mathrm{~nm}$ (Nd:YAG) pour éliminer les particules métalliques non expulsées par la vaporisation du lubrifiant [11].

On constate donc que le choix de la longueur d'onde repose sur une sélection du mode d'interaction en estimant dans ce cas les risques potentiels:

- soit de pollution organique par photodécomposition du lubrifiant (UV),

- soit d'endommagement possible du métal pour des énergies trop fortes $(>1 \mathrm{~J} / \mathrm{cm} 2)$ pour le procédé thermoélastique (IR).

Cependant en termes d'efficacité, l'utilisation d'un laser Nd:YAG (1.06 $\mu \mathrm{m})$ permet d'envisager l'utilisation de fibres optiques particulièrement intéressante dans le cas du nettoyage de tubes [12].

\section{CONCLUSION}

La nature des produits contaminants peut être minérale (oxydes, particules métalliques...) ou organiques (lubrifiants, polymères...) et leur absorption à la longueur d'onde du laser peut constituer un paramètre important pour optimiser l'efficacité du nettoyage.

Après avoir choisi les caractéristiques de l'émission laser, longueur d'onde et temps d'interaction en particulier, les exemples proposés montrent qu'il est également très important de définir les seuils d'énergie critiques qui conduisent au nettoyage sans altération fonctionnelle du substrat; cette notion d'altération fonctionnelle devant être définie en fait au cas par cas. Selon les systèmes, il s'agira du seuil d'ablation du substrat, du seuil de sa fusion superficielle, du seuil de sa mise en contrainte...

Le bref exposé des problèmes rencontrés illustre l'intérêt de développer des bases de données pour guider les choix des paramètres laser par l'utilisateur [13]. 


\section{Références}

[1] K.Mann, B.Wolff-Rottke, F.Müller, Applied Surface Science, 96-98, 463 (1996).

[2] W.Zapka, W.Zeimlich and A.C. Tam, Appl. Phys. Letters, 58, 2217 (1991).

[3] A.C. Engelsberg and J.A. Deshais, Advancing Microelectronics, 6 (1994),

[4] Y.F. Lu and Y. Aoyagi, Japan Journal of Applied Physics, 33, L430(1994).

[5] J.S. Foley and P.A.Barone, Metal Finishing, 48 (1992).

[6] Zapka, W Ziemlich, W P Leung and A C Tam, Microelectronic Engineering, 17 (1993).

[7] O.Yavas, A. Schilling, P. Leiderer, Applied Physics. A, Materials Science \& Processing, 64, 331 (1997).

[8] M.Mosbacher, V.Dobler, J.Boneberg, P.Leiderer, CLEO'98, Glasgow, 1998, paper CPD2.12

[9] Y.F. Lu, W.D. Song, M.H. Hong, B.S. Teo, T.C. Chong, T.S. Low, Journal of Applied Physics, 80, 499 (1996).

[10] R.Oltra, M. Indrianjafy, J.P. Boquillon, rapport interne Université de Bourgogne - Bourgogne Technologies, résultats non publiés.

[11] P.E. Lafargue, N. Chaoui, E.Millon, J.F. Muller, H.Derule, A.Popadenec, Surface and Coatings Technology, 106, 268 (1998).

[12] J.P. Boquillon, P. Bresson, H.Berger, Procédé de nettoyage de surface par laser impulsionnel, Brevet Français n8900496, Brevet USA n5151134, Brevet Européen 904001229.

[13] Projet TMR ERB FMRX-CT98-0188, Laser Cleaning, http://www.u-bourgogne.fr/REACTIVITE/TMR. 